Volume 9, No.4, July - August 2020

International Journal of Advanced Trends in Computer Science and Engineering

Available Online at http://www.warse.org/IJATCSE/static/pdf/file/ijatcse71942020.pdf

https://doi.org/10.30534/ijatcse/2020/71942020

\title{
A Hybrid Knowledge-Based and Collaborative Filtering Recommender System Model for Recommending Interventions to Improve Elderly Wellbeing
}

\author{
Aini Khairani Azmi ${ }^{1}$, Noraswaliza Abdullah ${ }^{2}$, Nurul A. Emran ${ }^{3}$ \\ ${ }^{1}$ Center for Advanced Computing Technology (C-ACT), Fakulti Teknologi Maklumat dan Komunikasi, \\ Universiti Teknikal Malaysia Melaka (UTeM), 76100 Durian Tunggal, Melaka, Malaysia, \\ aini.khairani@yahoo.com \\ ${ }^{2}$ Senior Lecturer, Center for Advanced Computing Technology (C-ACT), Fakulti Teknologi Maklumat dan \\ Komunikasi, Universiti Teknikal Malaysia Melaka (UTeM), 76100 Durian Tunggal, Melaka, Malaysia, \\ noraswaliza@utem.edu.my \\ ${ }^{3}$ Senior Lecturer, Center for Advanced Computing Technology (C-ACT), Fakulti Teknologi Maklumat dan \\ Komunikasi, Universiti Teknikal Malaysia Melaka (UTeM), 76100 Durian Tunggal, Melaka, Malaysia, \\ nurulakmar@utem.edu.my
}

\begin{abstract}
A recommender system is an information filtering system that helps users select items that most match their preferences from a vast amount of information available. It has been widely applied in many domains such as e-commerce, healthcare, entertainment and so on. Currently, there are some efforts have been done for recommending interventions to improve elderly well-being in different aspects of successful ageing. However, the recommendations are focused on only a single aspect of successful ageing such as nutrition or health. There are many aspects of successful ageing that should be considered when given interventions to improve elderly wellbeing namely socialization, health, physical, cognitive, nutrition, spirituality and environment. This paper aims to propose a Hybrid Knowledge-Based and Collaborative Filtering (KBCF) recommendation model to recommend interventions based on multiaspects of successful ageing in order to improve the elderly wellbeing. The Knowledge-based (KB) recommendations are generated by consulting a knowledge base which is developed based on knowledge provided by the domain experts. The Collaborative Filtering (CF) approach is applied to find similar users based on elderly profiles generated from the result of assessments done to the elderly. The results of the KB recommendations and the $\mathrm{CF}$ recommendations are integrated and ranked to select the final recommendations. The result of experiments conducted using precision, recall and F1 measure shows that the proposed KBCF model outperforms the baseline models which are Basic Search, Collaborative Filtering and Knowledge-based. This result demonstrates the proposed $\mathrm{KBCF}$ model provides more accurate and meaningful intervention recommendations for improving the elderly wellbeing in multiaspects of successful ageing.
\end{abstract}

Key words : Collaborative filtering, hybrid recommendation, knowledge-based, intervention recommendation.

\section{INTRODUCTION}

Nowadays more people living much longer lives with people above the age of 65 now make up an increasing share of the world's total population. Health care has become much more useful and treatment has advanced, thus allowing the elderly to live longer. In order to adapt to the increasingly aging populations, many countries have raised the retirement age, reduced pension benefits, and have started spending more on elderly care. Elderly in elderly care is expected to prolong significantly within the next 20 years. Individuals who need treatment will increase accordingly, while more individuals who can afford this treatment will be needed. Without receiving adequate care, the elderly people have the opportunity to lose their independence. Thereby, it is common for those who work at the Elderly Healthcare or caregivers to seek recommendations in ensuring that the elderly get their suitable treatments or interventions according to their achievement in varied aspects, such as health, physical, social, cognitive, spiritual, nutrition and environment.

The elderly people are most likely to age successfully with continuous monitoring and providing interventions for all of the successful ageing aspects. Therefore, a recommender system approach that consider the elderly conditions in many aspects of successful ageing is required to recommend interventions that can improve the elderly wellbeing. This research proposes a Hybrid Knowledge-Based and Collaborative Filtering (KBCF) recommender system model that can recommend interventions to the elderly by considering multiple aspects of successful ageing in order to help them achieve successful ageing. 


\section{RELATED WORKS}

A Collaborative Filtering (CF) is one of the most widely applied recommendation approach [1]. It is most successfully used for recommending frequently purchased or selected items such as movies, books and other e-commerce items. This approach requires a large amount of ratings data to make meaningful recommendations. It finds similar users based on ratings data on items purchased or selected by the users and recommends items to the active user based on items that have been purchased by similar users. However, this approach is not suitable for recommending interventions for elderly as a large amount of ratings data on interventions received by the elderly is not available.

Knowledge-based systems recommend items based on specific domain knowledge about how certain item features meet user's needs and preferences. A knowledge-based recommender system suggests items based on inferences about a user's needs and preferences. This knowledge will sometimes contain explicit functional knowledge about how certain items features meet user needs. In this approach, the functionality of item that meets the user requirement is important to recommend items that match with the user requirements. However, this approach requires an extensive knowledge engineering in making inference of the functionality of items towards the user requirement of the item. An example of knowledge-based recommender system is developed by [2] which is a mobile application of dietary nutrition recommendation service for obese management. In [3], a fuzzy-based recommender system is developed to address the issue of uncertainty in choosing the right career track by senior high school students.

A hybrid method was used because of the disadvantages that each of the recommender approaches have. Hybrid recommender system approach combines two or more recommender system approaches to improve the limitation of a single approach. For example, a hybrid system combining techniques A and B tries to use the advantages of $\mathrm{A}$ to fix the disadvantages of B. In [4], a combination of Knowledge-based using Ontology and sequential pattern mining with Collaborative filtering technique was proposed for e-learning recommendation. In [5], a hybrid of Content-based and Collaborative filtering techniques is used to recommend news in a way to have a tremendous impact on the user's mood. [6] applied Collaborative filtering and two (2) steps of algorithm which are clustering and classification for recommendation of beauty aesthetic services in a clinic.

Elderly observations using the recommender system are found to be implemented, but only focus on one aspect of successful ageing such as nutrition or health, which is not enough to help elderly to achieve successful ageing. There are some research works on recommender systems to improve elderly well-being have been conducted such as health recommendation [7],[8], [9] and nutritional and food recommendation [10], [4], [11]. However, there is no recommendation approach has been studied to recommend interventions for elderly based on multiaspects of successful ageing.

In this research, a Hybrid Knowledge-based and Collaborative Filtering (KBCF) model is proposed to recommend interventions for elderly by considering multiaspects of successful ageing based on user profiles generated from the assessment results of the elderly conditions. The detail methodology for this approach will be explained in the following section.

\section{METHODOLOGY}

Hybrid recommender systems combine two or more recommendation approaches to gain better performance with fewer of the drawbacks of any individual one. In this research, two recommendation approaches which are Collaborative Filtering and Knowledge-based Filtering will be combined. In the Collaborative Filtering approach, the interventions are recommended to the elderly based on the interventions given to the other elderly who have similar conditions with the elderly based on their profiles. The profile for each elderly is constructed from the assessment results performed to the elderly in different aspects of successful ageing such as health, physical, social, cognitive, spiritual, nutrition, and environment. Collaborative filtering technique uses k-NN method in which $\mathrm{k}$ number of users with similar profiles will be chosen as neighbors and the interventions that have been given to these users are recommended to the target user.

In the Knowledge-based filtering approach, the knowledge-based is constructed from knowledge gathered from the experts about which interventions should be recommended to the elderly based on their conditions. The knowledge base stores important rules that can be inferred by the recommender system for suggesting interventions that match with the user profiles. The result of the Collaborative Filtering and Knowledge-based recommendations are combined to generate more meaningful intervention recommendations. Next sections will explain about the user profile, Collaborative Filtering, Knowledge-based and the hybrid of Knowledge-based and Collaborative Filtering approaches in more details.

\subsection{User Profile}

The proposed recommender system must take the user assessment result for each aspect into consideration when making intervention recommendations for the elderly. A user profile for an elderly which represents the elderly conditions is constructed from the assessment result of each aspect. It consists of a vector of items and their ratings as shown below: 


$$
P_{i}=\left\{A_{i 2}, A_{i 2}, A_{i 2}, A_{i 4}, A_{i 5}, A_{i 6}, A_{i n}\right\}
$$

where $A$ is assessment aspect, $P$ is user profile and $i$ represents different users or elderly. There are seven (7) variables or aspects that present a user profile which are $A_{i 1}, A_{i 2} A_{i 3} A_{i 4}, A_{i 5} A_{i 6}$ and $A_{i z} . A_{i 1}$ represent the socialization aspect, $A_{i 2}$ represent the health aspect, $A_{i z}$ represent the cognitive aspect, $A_{\mathrm{IA}}$ represent the physical aspect, $A_{i E_{i}}$ represent the nutrition aspect, $A_{i 6_{i}}$ represent the spirituality aspect and $A_{\mathrm{i} \gamma_{2}}$ represent the environment aspect for user $i$. The method to calculate the value of each aspect in the user profile was discussed in our previous work [12].

\subsection{Collaborative Filtering Approach}

In this research, the k-Nearest Neighbor (k-NN) method and the cosine similarity method were used to implement neighborhood formation for the $\mathrm{CF}$ approach. The key technique of k-NN is to calculate the similarity between target user and other users, and then find the k nearest neighbors to predict suitable interventions for the target user. Cosine similarity method is used to find the similarity between the user's assessment profiles. In this case, two users were regarded as two vectors in the $\mathrm{n}$ dimensional item space. The similarity between them is measured by computing the cosine of the angle between these two vectors. Formally, similarity between users A and B is given as below where A and B represent the $n$ dimensional vectors those users accumulated on the $\mathrm{n}$ items.

$$
\operatorname{simi\operatorname {Tat}y(A,B)}=\frac{A \cdot B}{\|A\| \times\|\bar{B}\|}=\frac{\sum_{i=1} A_{i} \times B_{i}}{\sqrt{\sum_{i=1} A_{i}^{2}} \times \sqrt{\sum_{i=1} B_{i}^{2}}}
$$

In formula (2), $A$ represents the profile of an existing user while $B$ represents the profile of the target user. Each profile of user consists of seven (7) different values representing the result for each aspect of successful ageing. The Cosine Similarity values are calculated for other user profiles and the values are sorted to choose the selected (n) number of the most similar profiles. The number of neighbor that has been chosen for this experiment is five (5) because it achieved good results compared to other number of neighbors. Table 1 shows the algorithm of the $\mathrm{CF}$ approach.

Table 1: The Algorithm of the CF Recommendation Approach

Algorithm 3.1

Input:

A new user profile $\boldsymbol{U}_{i 1}$ and a set of user's neighbor

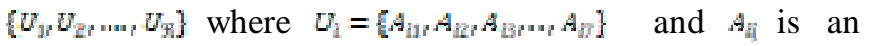
assessment value for each successful ageing aspect;

Intervention set $I_{U_{i}}=\left[T^{i 1}, T^{W}, \ldots, T^{E}\right]$ for each user $\bar{E}$ where

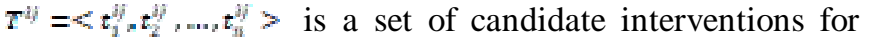
user and aspect $\bar{l}$;
Intervention candidate recommendations list

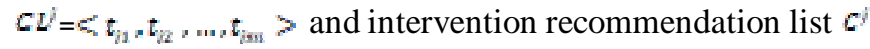
for each aspect $i$.

Output: A set of recommended interventions $\Gamma$

Method:

Begin

1. For each neighbour $u_{i}$ where $\mathrm{i}=1,2,3,4,5$

2. For each aspect $i$ where $\mathrm{j}=1,2,3,4,5,6,7$

3. For each intervention set $\boldsymbol{z}^{i j}$ in intervention set in $_{i}$

4. For each intervention $t_{i k}^{3 y}$

5. Check intervention $t_{i}^{i j}$ existence in candidate intervention recommendation list CL

6

If $t_{k}^{b} \in c \nu$

7. Calculate the frequency of the candidate intervention

8. recommendation $t_{\mathrm{m}}$, freq $_{\mathrm{m}}\left(\mathrm{t}_{\mathrm{m}}\right)_{+=1}$; Else

Assign intervention $t_{k}^{a j}$ into intervention candidate list $\mathrm{Cs}^{i}<-t_{i}^{3 j}$

9. For each candidate intervention recommendation list $\mathrm{CL}$

10. Sort intervention $t_{\text {in }}$ based on the freq $\left(t_{i n}\right)$ value

11. Select top $\mathrm{N}$ interventions $\tau_{\bar{m} m}$ and assign into $c^{i}$

12. Return $\Gamma_{i}=U_{j=1}^{7} C^{\prime}$

End

\subsection{Knowledge-based Approach}

A knowledge-based approach exploits knowledge of the item domain and the user requirements in reasoning what items meet the user requirements. Rule-based system is used as a way to store and manipulate knowledge to interpret information in a useful way. In this research, the rules for intervention recommendations are generated based on knowledge gathered from the domain experts who in charge of the elderly's health, physical, social, cognitive, spiritual, nutrition and environment aspects. For using rule-based technique, user preferences of the intervention recommendations need to be identified. The user preferences or profiles are generated from the results of assessments conducted to the elderly on each aspect of successful ageing. These user profiles are used to classify the elderly into a category of successful, semi successful or non-successful ageing. Based on the classified group of the elderly, the knowledge-based is consulted to recommend interventions to the elderly by using the rules stored in the knowledge base. 


\subsection{Hybrid of Knowledge-based and Collaborative Filtering Approaches}

In the Hybrid Knowledge-based and Collaborative Filtering (KBCF) approach, the results of Collaborative Filtering and Knowledge-based approaches are combined and the five most frequent interventions for each aspect are selected to be recommended to the elderly. Figure 1 shows the framework of the Hybrid of Knowledge-based and Collaborative filtering (KBCF) approach.

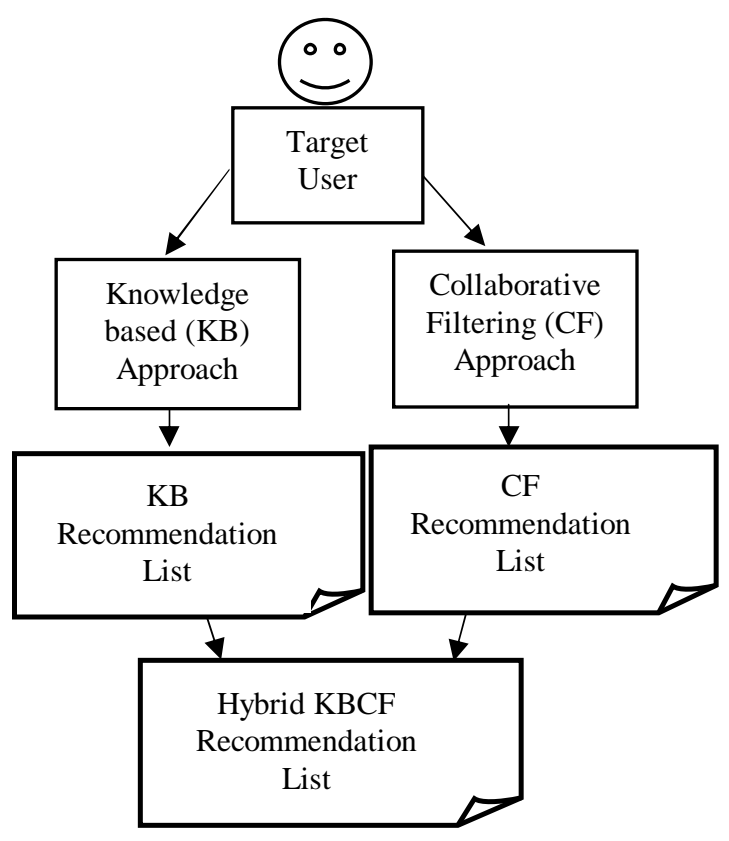

Figure 1: The framework of the Hybrid Knowledge-based and Collaborative Filtering (KBCF) approach

\section{EXPERIMENT}

The experiments were conducted to see how the proposed Hybrid Knowledge-based and Collaborative Filtering (KBCF) model performs compared to the baseline models. The experiments were conducted based on the following hypothesis:

Hypothesis: The proposed hybrid recommender system model which integrates Knowledge-based and Collaborative Filtering approaches can generate more accurate recommendations compared to the baseline models such as Basic Search, Collaborative Filtering and Knowledge-based.

\subsection{Dataset}

The data used in this research was an assessment data for seven (7) aspects of successful ageing collected from assessments conducted by experts to the elderly on their health, social, physical, nutrition, spiritual, cognitive and environment aspects. The intervention data was also collected from the interventions suggested by the experts based on the elderly assessment results. There were 139 samples of users involved in this research (elderly aged above 60 living in an elderly institution). For each elderly, data about assessment results conducted on them and interventions given to them by the experts based on their assessment results were collected. Therefore, there were 139 user profiles were created to be used by the Collaborative Filtering for neighborhood formation and intervention recommendations. These profiles are also used by the Knowledge-based approach to consult the knowledge base in order to recommend interventions that match with the elderly conditions.

\subsection{Evaluation Metrics}

An evaluation of the proposed Hybrid Knowledge-based and Collaborative Filtering (KBCF) recommender system model was made to measure the levels of their accuracy and to assess which of the recommendation model performs the best. In this experiment, precision, recall and F1 Measurement metrics were used to evaluate the accuracy of the proposed models.

- $\quad$ Recall and Precision

Recall and Precision are the measures for measuring the efficiency and the suitability of the recommendation obtained by the recommender system. Recall measures the ability of the system to present all the relevant items and it can be seen as the measure of completeness. Recall also refers to the percentages of the relevant recommendation that were retrieved, out of the total relevant recommendations.

$$
\text { Recall }=\frac{N M}{N T}
$$

Precision measures the ability of the system to present only those items that are relevant, and it can be seen as the measure of exactness. It is also referring to the percentages of the relevant recommendation that were retrieved, out of the total retrieved recommendations.

$$
\text { Frecision }=\frac{N M}{N R}
$$

In this research, the evaluation was performed by considering $\mathrm{NM}$ as the number of suggested interventions matching the testing interventions, while NT as the number of interventions that should be returned and NR is the number of returned suggested interventions.

- $\quad$ F1 Measure

The F1 metric was used to provide a general overview of the overall performance. The F1 measure combined the recall and precision results with an equal weight in the following form:

$$
f 1=\frac{2 \times \text { Precision } \times \text { Recall }}{\text { Precision }+ \text { Recall }}
$$




\subsection{Experimental Setup}

In order to evaluate the accuracy of the proposed Hybrid Knowledge-based and Collaborative Filtering (KBCF) recommendation model, this paper implemented the user profiling, the proposed KBCF recommendation model and the baseline models. For this experiment, 139 profiles of users were used as testing and training datasets. There were three runs of experiment conducted and the 139 profiles were divided into 3 groups (A, B and C). The division of dataset contained two (2) parts which were Training and Testing as illustrated in Figure 2, 3 and 4 below:

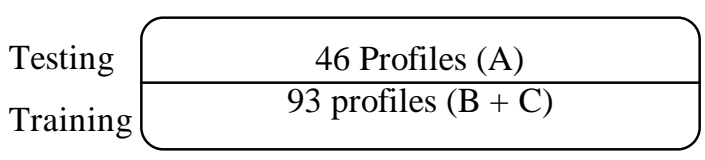

Figure 2: The division of dataset for the experiment (Run 1)

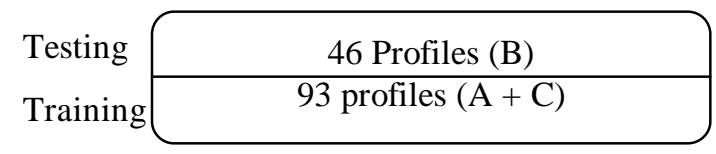

Figure 3: The division of dataset for the experiment (Run 2)

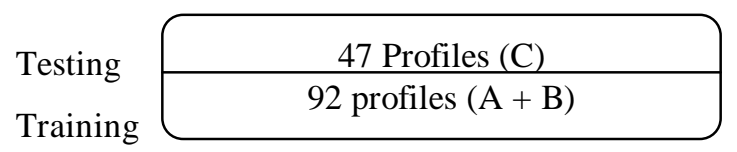

Figure 4: The division of dataset for the experiment (Run 3)

The testing dataset was considered as the target users or new users while the training dataset was considered as previous users. Training dataset was used to generate previous users' profiles which were used to find neighbors by using the neighborhood formation method. The interventions for neighborhood users were used to recommend interventions for a new user. The precision, recall and F1 results for each run were calculated. Finally, the average result for each recall, precision and F1 Measure of the three (3) runs was calculated. The experiments were conducted to test if the proposed model which is the Hybrid Knowledge-based and Collaborative Filtering (KBCF) was able to suggest more accurate interventions compared to baseline models which are Basic Search (BS), Collaborative Filtering (CF) and Knowledge based $(\mathrm{KB})$. The description of each model is explained as follows:

- Basic Search (BS): This model produced recommendations based on the similarity of the target user profile with the most similar user by matching the value of each aspect of successful ageing in the target user profile with the value of each aspect of successful ageing of other users.
- Collaborative Filtering (CF): This model produced recommendations based on interventions given to the top five (5) similar users of the target user that were identified based on the user profiles of target user and other users.

- Knowledge-based (KB): This model produced recommendations by consulting the knowledge-base of interventions gathered from the experts which was stored as rules in the knowledge-base.

- Hybrid Knowledge-based and Collaborative Filtering (KBCF): This model produced recommendations by combining recommendations produced by the Knowledge-based and Collaborative Filtering models. The recommendations are selected based on the interventions that are mostly or frequently produced by both models.

\section{RESULT AND DISCUSSION}

The objective of this set of experiments is to verify that the Hybrid Knowledge-based and Collaborative Filtering (KBCF) model can generate more accurate recommendations compared to Basic Search (BS), Collaborative Filtering (CF) and Knowledge-based (KB).

Table 2: The results of Precision, Recall and F1 Measure

\begin{tabular}{|l|l|l|l|}
\hline Methods & Precision & Recall & F1 Measure \\
\hline Basic Search (BS) & 0.482 & 0.489 & 0.485 \\
\hline $\begin{array}{l}\text { Collaborative } \\
\text { Filtering (CF) }\end{array}$ & 0.589 & 0.503 & 0.543 \\
\hline $\begin{array}{l}\text { Knowledge-based } \\
\text { (KB) }\end{array}$ & 0.662 & 0.491 & 0.564 \\
\hline $\begin{array}{l}\text { Hybrid } \\
\text { Knowledge-based } \\
\text { and Collaborative } \\
\text { Filtering (KBCF) }\end{array}$ & 0.785 & 0.503 & 0.613 \\
\hline
\end{tabular}

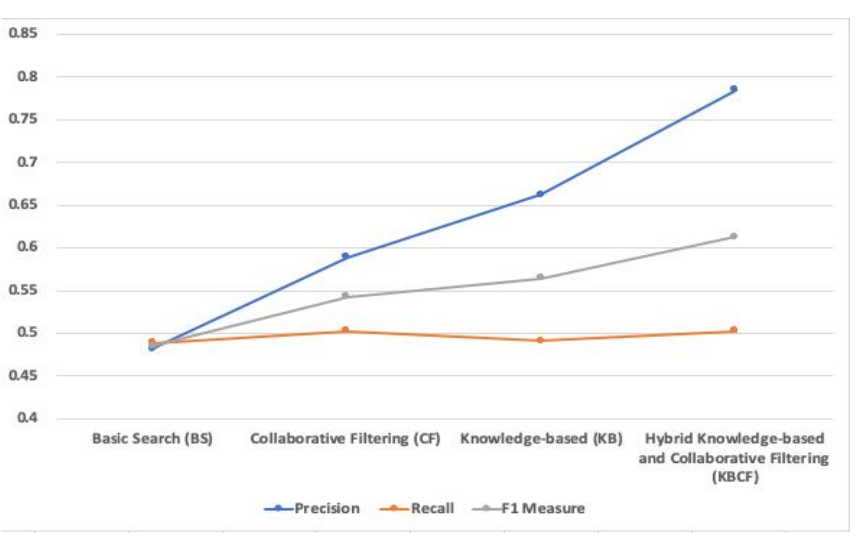

Figure 5: Precision, Recall and F1 Measure results for BS, $\mathrm{CF}, \mathrm{KB}$ and $\mathrm{KBCF}$ methods 
Table 2 and Figure 5 shows the results of precision, recall and F1 Measure for the proposed model and the baseline models. Basic Search (BS) has the lowest precision, recall and F1 measure compared to the other models. The precision, recall and F1 Measure results for the Collaborative Filtering (CF) were higher than the BS. However, the precision and F1 Measure results for the $\mathrm{CF}$ were lower than $\mathrm{KB}$ and KBCF. In addition, the recall result of $\mathrm{CF}$ is better than $\mathrm{KB}$ and the same as $\mathrm{KBCF}$. The precision and F1 Measure results for the Knowledge-based (KB) are higher than BS and CF. However, the recall for $\mathrm{KB}$ is lower than $\mathrm{CF}$ but higher than BS. The $\mathrm{KBCF}$ has the highest precision and F1 Measure results among all the models. The recall result of KBCF is higher than BS and KB but the same as CF. This result shows the Hybrid Knowledge-based and Collaborative Filtering (KBCF) model outperforms other baseline models that apply only a single recommendation approach.

This result shows that by integrating $\mathrm{CF}$ and $\mathrm{KB}$ approaches, more accurate intervention recommendations can be generated to the users compared to using a single approach. When CF is applied, the recall result of the method increases. This shows that $\mathrm{CF}$ approach generates more interventions that match with the user's profile or condition. This approach recommends more matching interventions based on interventions suggested for the users with similar profiles or conditions. On the other hand, the precision of the method increases when $\mathrm{KB}$ is applied. This shows that more interventions recommended by the $\mathrm{KB}$ are match with the target user profile because recommendations given by this approach are based on the recommendations given by the experts. Thus, the integration of Knowledge-based and Collaborative Filtering (KBCF) approaches recommend more complete interventions that highly match with the target user's profile or condition.

\section{FUTURE WORKS}

This research work involves a small set of data because the user profiles are generated from the results of assessment which are conducted to the elderly only twice a year. This research could be extended by collecting more users' assessment results to generate more user profiles. Clustering technique can be applied to group the elderly based on their profiles. The neighbors of the users can be selected from the same user clusters. This technique may improve the accuracy and performance of the proposed model.

\section{CONCLUSION}

This research aims to recommend interventions for improving elderly well-being in multiaspects of successful ageing namely health, physical, social, cognitive, spiritual, nutrition, and environment. A Hybrid Knowledge-based and Collaborative Filtering recommendation approach (KBCF) is proposed to recommend interventions for all of these aspects.
The KBCF approach utilizes user profiles that are generated from the assessment results of elderly conditions in those aspects. Experiment results show that the proposed Hybrid Knowledge-based and Collaborative Filtering model outperforms baseline models such as Basic Search (BS), Collaborative Filtering (CF) and Knowledge-based (KB). The results reveal that the proposed model is able to recommend accurate interventions for improving elderly well-being in multiaspects of successful ageing. By implementing the proposed model, caregivers can perform the recommended interventions to the elderly without waiting for the experts to evaluate the elderly conditions and provide intervention suggestions. The interventions performed on many aspects of successful ageing may improve the elderly well-being and help them to achieve successful ageing.

\section{ACKNOWLEDGEMENT}

The authors would like to thank the Center of Advanced Computing Technologies (CACT), Fakulti Teknologi Maklumat dan Komunikasi, Universiti Teknikal Malaysia Melaka (UTeM), 76100 Durian Tunggal, Melaka, Malaysia for supporting this research.

\section{REFERENCES}

1. R. Chen, Q. Hua, Y. Chang, B. Wang, L. Zhang, and X. Kong. A survey of Collaborative Filtering-based recommender systems: from traditional methods to hybrid methods based on social networks, IEEE Access, vol. 6, pp. 64301-64320, 2018. https://doi.org/10.1109/ACCESS.2018.2877208

2. H. Jung and K. Chung. Knowledge-based dietary nutrition recommendation for obese management, Information Technology and Management, vol 17, no. 1, pp. 29-42, 2016.

3. M. C. B. Natividad, B. D. Gerardo and R. P. Medina. A Carrer Track Recommender System for Senior High School Students using Fuzzy Logic,. International Journal of Advanced Trends in Computer Science and Engineering, vol. 8, no. 5, pp. 2512-2519.

https://doi.org/10.30534/ijatcse/2019/97852019

4. J. K. Tarus, Z. Niu and A. Yousif. A hybrid knowledge-based recommender system for e-learning based on ontology and sequential pattern mining, Future Generation Computer Systems, vol. 72, pp. 37-48, 2017.

5. A. H. Parizi and M. Kazemifard. Emotional news recommender system, in 2015 Sixth International Conference of Cognitive Science (ICCS), Tehran, 2015, pp. 37-41.

https://doi.org/10.1109/COGSCI.2015.7426666

6. F. Pemisindo and N. Legowo. Hybrid Recommendation System with Clustering and Classification Method Based on Case of Clinic XYZ. International Journal of Advanced Trends in Computer Science and Engineering, vol. 9, pp. 1352-1357, 2020.

https://doi.org/10.30534/ijatcse/2020/69922020 
7. C. L. S. Bocanegra, J. L. S. Ramos, C. Rizo, A. Civit and L. Fernandez-Luque. HealthRecSys: A semantic content-based recommender system to complement health videos. BMC Medical Informatics and Decision Making, vol. 17, no. 1, 2017.

8. L.R. Ferretto, C. R. Cervi and A. C. B. de Marchi Recommender systems in mobile apps for health a systematic review, in 12th Iberian Conference on Information Systems and Technologies (CISTI), 2017, pp.1-6.

9. S. S. A. Naser and S. H. ALmursheidi. A Knowledge Based System for Neck Pain Diagnosis. World Wide Journal of Multidisciplinary Research and Development (WWJMRD), vol. 2, no. 4, pp. 12-18, 2016.

10. D. Ribeiro, J. Machado, J. Ribeiro, M. J. M. Vasconcelos, E. F. Vieira and A. C. de Barros. SousChef: Mobile Meal Recommender System for Older Adults, in 3rd International Conference on Information and Communication Technologies for Ageing Well and e-Health, 2017, pp. 36-45. https://doi.org/10.5220/0006281900360045

11. G. Agapito, B. Calabrese, P. H. Guzzi, M. Cannataro, M. Simeoni, I. Caré... and A. Pujia. DIETOS: a recommender system for adaptive diet monitoring and personalized food suggestion. In 12th International Conference on Wireless and Mobile Computing, Networking and Communications (WiMob), 2016, pp. 1-8.

12. A. K. Azmi., N. Abdullah and N. A Emran. A Collaborative Filtering recommender system model for recommending intervention to improve elderly well-being. International Journal of Advanced Computer Science and Applications(IJACSA), vol. 10, no. 6, pp.131-138, 2019.

https://doi.org/10.14569/IJACSA.2019.0100619 